\title{
Quarterly Models Of Intercity Freight Demand
}

\author{
H. Wade German* and Mary D. Sianis**
}

INTRODUCTION

Every industry and firm must have a flow of information concerning expected demand for its product or service. The interdependent nature of the transportation industry in general and the rail industry in particular creates a crucial need for accurate forecasts of modal transportation demand. For an industry in which intermodal shipments are increasing as a percentage of total traffic and, therefore, the economic health of one mode is partially dependent upon other modes, both motor carrier and railroad executives are increasingly called upon to evaluate the outlook for the entire industry as opposed to just their particular mode and company. In this type of operating environment, there is no substitute for a national transportation forecasting system which translates movements in the aggregate industry indicators into forecasts for various modes on a commodity basis as well as equipment requirements.

In reviewing the literature on freight demand forecasting, it is apparent that a disproportionate share of effort by economists in this area has been directed toward the identification and measurement of "modal split determinants"; i.e., previous research has been focused almost exclusively on "internal" industry economics. Although there is little doubt that cross price elasticity of demand is of paramount importance in the competitive freight transportation environment, it is likewise important not to lose sight of those forces external to the freight sector which generate the "derived" demand for transportation services.

Another major shortcoming in previous studies is the tendency to analyze annual data since it is generally more readily available. The use of annual data, however, obliterates all the seasonality of freight demand patterns and much of the cyclical aspects as well. Additionally, both transportation firms as well as equipment manufacturers schedule equipment, material purchases and labor availability on a quarterly or monthly basis and, hence, annual forecasts of demand are of little use in the short term planning requirements of most firms.

This paper bridges two gaps relative to the transportation industry's information needs. First, we have focused on the linkages between the aggregate level of economic activity and intercity freight demand and,

*Director of Commercial Systems and Quantitative Analysis, Union Pacific Railroad

**Senior Economic Analyst, Union Pacific Railroad. 
secondly, we have utilized quarterly data to provide a more direct input into the short term decision-making process at the industry and firm level.

\section{THE COMPOSITION OF ECONOMIC ACTIVITY AND TRANSPORTATION DEMAND}

Over the post war time period, economic activity as measured by real Gross National Product has advanced 104 percent (see Table 1) from 655 billion in 1955 to $\$ 1333$ billion in 1977 . In the same time interval, industrial production rose 135 percent and total intercity freight ton miles registered a more modest 83 percent gain. Hence, it is clear that there is not a one-to-one correspondence between economic activity and transportation demand. This is due principally to the change in the composition of economic activity over time. ${ }^{1}$ An example of this is the rapid growth of the services component of personal consumption outlays-a sector of GNP which generates very little, if any, freight demand. In 1955, the services component of personal consumption accounted for 39.9 percent of total

TABLE 1

The Performance of the Transportation Sector

Relative to Economic Growth

1955-1977

\begin{tabular}{|c|c|c|c|c|c|c|}
\hline \multirow[b]{2}{*}{ Year } & \multirow[b]{2}{*}{$G_{N P}{ }^{1}$} & \multicolumn{2}{|c|}{ Real Consumption } & \multirow{2}{*}{$\begin{array}{c}\text { xpenditures }^{1} \\
\text { Services } \\
\text { As \% Total }\end{array}$} & \multirow{2}{*}{$\begin{array}{c}\text { Industrial }^{2} \\
\text { Production } \\
(1967=100.0)\end{array}$} & \multirow{2}{*}{$\begin{array}{c}\text { Intercity } \\
\text { Freight Ton } \\
\text { Miles } \\
\text { (Billions) }\end{array}$} \\
\hline & & Total & Services & & & \\
\hline 1955 & 654.8 & 395.5 & 157.5 & 39.9 & 58.4 & 1,274 \\
\hline 1960 & 736.8 & 453.0 & 192.3 & 42.5 & 66.1 & 1,314 \\
\hline 1961 & 755.3 & 462.2 & 200.0 & 43.3 & 66.6 & 1,310 \\
\hline 1962 & 799.1 & 482.9 & 208.7 & 43.2 & 72.1 & 1,371 \\
\hline 1963 & 830.7 & 501.4 & 217.6 & 43.4 & 76.6 & 1,453 \\
\hline 1964 & 874.4 & 528.7 & 229.7 & 43.4 & 81.7 & 1,543 \\
\hline 1965 & 925.9 & 558.1 & 240.7 & 43.1 & 89.8 & 1,638 \\
\hline 1966 & 981.0 & 586.1 & 251.6 & 42.9 & 97.7 & 1,747 \\
\hline 1967 & 1007.7 & 603.2 & 264.0 & 43.8 & 100.0 & 1,765 \\
\hline 1968 & 1051.8 & 633.4 & 275.0 & 43.4 & 106.3 & 1,838 \\
\hline 1969 & 1078.8 & 655.4 & 287.2 & 43.8 & 111.2 & 1,895 \\
\hline 1970 & 1075.3 & 668.9 & 297.3 & 44.4 & 107.8 & 1,936 \\
\hline 1971 & 1107.5 & 691.9 & 306.3 & 44.3 & 109.6 & 1,954 \\
\hline 1972 & 1171.1 & 733.0 & 322.4 & 44.0 & 119.7 & 2,073 \\
\hline 1973 & 1235.0 & 767.7 & 336.5 & 43.8 & 129.7 & 2,232 \\
\hline 1974 & 1217.8 & 760.7 & 344.3 & 45.3 & 129.3 & 2,212 \\
\hline 1975 & 1202.3 & 774.6 & 355.3 & 45.9 & 117.8 & 2,066 \\
\hline 1976 & 1271.0 & 819.4 & 373.2 & 45.5 & 129.8 & 2,200 \\
\hline 1977 & 1332.7 & 857.7 & 389.5 & 45.4 & 137.1 & 2,331 \\
\hline \multicolumn{7}{|c|}{ \% Change } \\
\hline $1955-77$ & 104 & 117 & 147 & & 135 & 83 \\
\hline
\end{tabular}

Billions of 1972 dollars.

${ }^{2}$ Revised index only available from 1954. 
consumption compared to 45.4 percent in 1977 . Stated alternatively, the output of "transportable" goods has lagged behind the overall rate of economic growth.

As noted in Table 2, all transport modes have not received proportional shares of the expanding freight market. The lion's share of the expanding "freight pie" accrued to inland water carriers who experienced a 439 percent leap in ton miles followed by motor carriers with a 224 percent gain in freight ton miles. Class 1 and 2 Railroads registered a modest 39 percent increase in industry output in this time interval.

The percentage change in alternative market share measures for the railroad industry and regulated motor carriers is presented in Table 3.

\section{QUARTERLY DEMAND FOR RAIL AND MOTOR CARRIER SERVICES}

Quarterly data availability for the rail industry as a whole consists of net revenue ton miles and freight carloadings-both published by Association of American Railroads. However, each individual railroad must submit a Quarterly Commodity Statistics (QCS). Report to the Interstate Commerce Commission and, therefore, quarterly tonnage, carloading and revenue

TABLE 2

Intercity Freight Ton Miles By Mode Selected Years: 1950-1977

(Billions of Ton Miles)

\begin{tabular}{cccccccc}
\hline Year & $\begin{array}{c}\text { Class 1 and 2 } \\
\text { Railroads }\end{array}$ & $\begin{array}{c}\text { Motor } \\
\text { Carriers }\end{array}$ & $\begin{array}{c}\text { Great } \\
\text { Lakes }\end{array}$ & $\begin{array}{c}\text { Rivers and } \\
\text { Canals }\end{array}$ & $\begin{array}{c}\text { Oil } \\
\text { Pipeline }\end{array}$ & Airlines & Total \\
\hline 1950 & 597 & 173 & 112 & 52 & 129 & 0.318 & 1,063 \\
1955 & 631 & 223 & 119 & 98 & 203 & 0.481 & 1,274 \\
1960 & 579 & 298 & 99 & 121 & 229 & 0.778 & 1,314 \\
1965 & 709 & 371 & 110 & 152 & 310 & 1.910 & 1,638 \\
1966 & 751 & 381 & 116 & 164 & 333 & 2.250 & 1,747 \\
1967 & 731 & 389 & 107 & 174 & 361 & 2.592 & 1,765 \\
1968 & 757 & 396 & 112 & 179 & 391 & 2.900 & 1,838 \\
1969 & 780 & 404 & 115 & 188 & 411 & 3.200 & 1,895 \\
1970 & 771 & 412 & 114 & 205 & 341 & 3.400 & 1,936 \\
1971 & 746 & 430 & 105 & 210 & 444 & 3.400 & 1,954 \\
1972 & 784 & 470 & 109 & 230 & 480 & 3.700 & 2,073 \\
1973 & 858 & 505 & 126 & 232 & 507 & 3.945 & 2,232 \\
1974 & 852 & 495 & 107 & 248 & 506 & 3.910 & 2,212 \\
1975 & 759 & 454 & 99 & 243 & 507 & 3.730 & 2,066 \\
1976 & 799 & 510 & 106 & 267 & 523 & 3.900. & 2,209 \\
1977 & 831 & 561 & 95 & 280 & 560 & 4.180 & 2,331 \\
$\%$ Change & & & & & & & \\
$1950-77$ & 39.2 & 224.3 & -15.2 & 438.5 & 334.1 & 1215. & 154.5 \\
\hline
\end{tabular}

Source:

Transportation Association of America, Transportation Facts and Trends-1978 (Washington, D.C.) 
data can be obtained in five-digit Standard Transportation Commodity Code configuration by aggregating individual firm data to a national total.

For the motor carrier industry, there are three principal data sources for quarterly demand patterns. First, the Commerce Department's Current Industrial Report (M37-L) for truck trailers reports highway truck trailer production-by type of trailer-on a monthly basis. Secondly, the Motor Vehicle Manufacturers Association (MVMA) reports on a monthly basis retail sales-by truck size-by manufacturer. Third, the American Trucking Association (ATA) compiles a monthly index of truck tonnage which measures the volume of general freight transported by Class 1 and 2 Motor Carriers. Since both truck trailers and power units are placed into immediate service, either measure is a good barometer of the demand for motor carrier transportation. The ATA truck tonnage index is deficient in that general freight is a declining proportion of total motor carrier tonnage and, therefore, understates the overall demand for motor carrier services. Hence, the quarterly freight carloadings data for the rail industry are comparable to the retail sales data for heavy duty trucks.

Historical data on freight carloadings and retail sales of heavy duty trucks over the 1960-1977 time period are detailed in Tables 4 and 5. Since

\section{TABLE 3}

Summary of Market Share Measures for

Class 1 \& 2 Railroads and Regulated

Motor Carriers: 1950-1978

\begin{tabular}{|c|c|c|c|c|c|c|}
\hline \multirow[b]{2}{*}{ Year } & \multicolumn{3}{|c|}{ Class 1 \& 2 Railroads } & \multicolumn{3}{|c|}{$\begin{array}{c}\text { Regulated } \\
\text { Motor Carriers }\end{array}$} \\
\hline & Tonnage & Ton Miles & Revenue & Tonnage & Ton Miles & Revenue \\
\hline 1950 & 46.7 & 56.2 & 63.4 & 7.0 & 16.3 & 26.9 \\
\hline 1955 & 40.9 & 49.5 & 56.0 & 8.8 & 17.5 & 32.5 \\
\hline 1960 & 36.1 & 44.1 & 49.0 & 10.7 & 21.8 & 39.1 \\
\hline 1965 & 3.3 .3 & 43.3 & 43.8 & 12.6 & 21.9 & 44.5 \\
\hline 1966 & 33.1 & 43.0 & 43.1 & 12.9 & 21.8 & 45.9 \\
\hline 1967 & 31.3 & 41.4 & 42.7 & 12.5 & 22.0 & 46.4 \\
\hline 1968 & 31.3 & 41.2 & 41.4 & 13.2 & 21.5 & 46.7 \\
\hline 1969 & 31.3 & 40.8 & 40.1 & 13.8 & 21.3 & 48.1 \\
\hline 1970 & 31.1 & 39.7 & 39.8 & 13.1 & 21.3 & 48.9 \\
\hline 1971 & 29.4 & 38.2 & 39.9 & 14.1 & 22.8 & 48.9 \\
\hline 1972 & 29.3 & 37.7 & 39.3 & 14.7 & 22.7 & 49.3 \\
\hline 1973 & 29.7 & 38.5 & 38.7 & 15.2 & 22.6 & 50.0 \\
\hline 1974 & 30.3 & 38.6 & 38.0 & 14.9 & 22.4 & 50.9 \\
\hline 1975 & 29.6 & 36.7 & 38.8 & 14.4 & 22.0 & 49.1 \\
\hline 1976 & 28.0 & 36.3 & 36.1 & 15.3 & 23.2 & 52.1 \\
\hline 1977 & 26.6 & 36.0 & 33.9 & 16.0 & 24.0 & 54.5 \\
\hline 1978 & 26.0 & 35.8 & $\mathrm{~N} / \mathrm{A}$ & 16.3 & 24.7 & $\mathrm{~N} / \mathrm{A}$ \\
\hline \multicolumn{7}{|l|}{$\%$ Change } \\
\hline $1950-78$ & -44.3 & -36.3 & -46.5 & 132.9 & 51.5 & 102.6 \\
\hline
\end{tabular}

Source: Transportation Association of America, Transportation Facts and Trends-1979. 
Historical Freight

Car Loadings

(Unadjusted)

\begin{tabular}{ccccc}
\hline Year & 1st Qtr. & 2nd Qtr. & 3rd Qtr. & 4th Qtr. \\
\hline 1960 & 7,576 & 8,113 & $-7,590$ & 7,161 \\
1961 & 6,384 & 7,209 & 7,490 & 7,505 \\
1962 & 6,902 & 7,473 & 7,195 & 7,151 \\
1963 & 6,599 & 7,604 & 7,319 & 7,348 \\
1964 & 6,794 & 7,518 & 7,436 & 7,688 \\
1965 & 6,862 & 7,543 & 7,416 & 7,425 \\
1966 & 6,989 & 7,628 & 7,522 & 7,482 \\
1967 & 6,763 & 7,239 & 6,976 & 7,104 \\
1968 & 6,651 & 7,371 & 7,154 & 7,075 \\
1969 & 6,571 & 7,300 & 7,114 & 7,250 \\
1970 & 6,450 & 7,173 & 6,823 & 6,713 \\
1971 & 6,445 & 6,816 & 6,214 & 5,788 \\
1972 & 6,148 & 6,661 & 6,568 & 6,725 \\
1973 & 6,558 & 6,980 & 6,902 & 6,896 \\
1974 & 6,596 & 6,860 & 6,538 & 6,187 \\
1975 & 5,667 & 5,895 & 5,735 & 5,928 \\
1976 & 5,731 & 6,094 & 5,878 & 5,751 \\
1977 & 5,610 & 6,224 & 5,845 & 5,616 \\
\hline
\end{tabular}

Source: Association of American Railroads, Statistics of Railroads of Class 1-Selected Issues.

TABLE 5

Historical Retail Unit

Sales of Heavy

Duty Trucks

(Unadjusted)

\begin{tabular}{rrrrr}
\hline Year & 1st Qtr. & 2nd Qtr. & 3rd Qtr. & 4th Qtr. \\
\hline 1960 & 8,347 & 10,259 & 6,436 & 5,146 \\
1961 & 5,789 & 8,685 & 6,674 & 6,207 \\
1962 & 8,321 & 12,032 & 10,605 & 9,661 \\
1963 & 11,389 & 15,002 & 11,613 & 12,050 \\
1964 & 15,255 & 18,476 & 13,687 & 11,882 \\
1965 & 16,036 & 18,985 & 17,058 & 16,510 \\
1966 & 21,103 & 23,282 & 19,645 & 19,789 \\
1967 & 20,018 & 20,962 & 15,740 & 15,559 \\
1968 & 18,656 & 22,081 & 19,181 & 20,026 \\
1969 & 24,246 & 27,654 & 24,069 & 23,064 \\
1970 & 24,020 & 25,308 & 20,484 & 21,260 \\
1971 & 22,819 & 26,763 & 24,285 & 24,797 \\
1972 & 28,181 & 33,199 & 30,851 & 34,064 \\
1973 & 36,935 & 42,426 & 36,698 & 38,512 \\
1974 & 38,364 & 42,079 & 36,953 & 30,137 \\
1975 & 24,053 & 21,284 & 18,911 & 18,900 \\
1976 & 19,508 & 24,288 & 27,604 & 25,886 \\
1977 & 32,838 & 39,826 & 34,080 & 33,899 \\
\hline
\end{tabular}

Source: Motor Vehicle Manufacturers Association of the U.S., Total New Truck Retail Sales and Stock-Selected Issues. 
the average capacity of the rail car fleet has increased significantly over the historical time period due to technological improvements, it is necessary to adjust the historical data in Table 4 by the index of capacity in Table 6 to derive a consistent set of demand estimates over time.

There is a strong seasonal pattern-as evidenced by Tables 7 and 8 -for both freight carloadings and retail sales of Class 8 heavy duty trucks. Tables 9 and 10 contain the quarterly seasonal adjustment factors for rail carloadings and retail truck sales for selected years.

\section{THE ECONOMY - RAIL AND \\ TRUCK INDUSTRY LINKAGES}

To accurately forecast intercity freight demand on a modal basis, it is necessary to know the commodity mix transported by each mode. The historical commodity mix of Class 1 Railroads is outlined in Table 13 with similar data for Class 1 and 2 Motor Carriers presented in Table 14.

The mix of commodities transported on the nation's rail network has remained relatively stable overall, with Chemical Products (STCC 28), Transportation Equipment (STCC 37), and Merchandise Traffic (STCCs 44, 45 and 46) experiencing growth and declines concentrated principally in manufactured goods. As indicated in Table 14, tonnage originated by Class 1 and 2 Motor Carriers has advanced at a rapid pace, with total

TABLE 6

Average Capacity Per

Freight Car (Tons)

\begin{tabular}{ccc}
\hline Year & & $\begin{array}{c}\text { Index } \\
(1960=1.00)\end{array}$ \\
\hline 1960 & Tons Per Car & 1.00 \\
1961 & 55.4 & 1.01 \\
1962 & 55.7 & 1.02 \\
1963 & 56.3 & 1.03 \\
1964 & 56.8 & 1.05 \\
1965 & 58.2 & 1.08 \\
1966 & 59.7 & 1.11 \\
1967 & 61.4 & 1.14 \\
1968 & 63.4 & 1.16 \\
1969 & 64.3 & 1.19 \\
1970 & 65.8 & 1.21 \\
1971 & 67.1 & 1.23 \\
1972 & 68.4 & 1.26 \\
1973 & 69.6 & 1.27 \\
1974 & 70.5 & 1.29 \\
1975 & 71.6 & 1.32 \\
1976 & 72.9 & 1.33 \\
1977 & 73.8 & 1.36 \\
\hline
\end{tabular}

Source: Statistics of Railroads of Class 1-Selected Issues 
TABLE 7

Predictable Seasonality Test

Freight Car Loadings

\begin{tabular}{lccrc}
\hline & $\begin{array}{c}\text { Sum of } \\
\text { Squares }\end{array}$ & $\begin{array}{c}\text { Degrees of } \\
\text { Freedom }\end{array}$ & $\begin{array}{c}\text { Mean } \\
\text { Square }\end{array}$ & F Value \\
\hline Between Quarters & 650.361 & 3 & 216.787 & $93.459^{*}$ \\
Residual & 157.733 & 68 & 2.320 & \\
Total & 808.094 & 71 & & \\
\hline
\end{tabular}

*Predictable seasonality present at the 99.99 percent confidence level.

TABLE 8

Predictable Seasonality Test

Retail Sales of Heavy

Duty Trucks

\begin{tabular}{lcccc}
\hline & $\begin{array}{c}\text { Sum of } \\
\text { Squares }\end{array}$ & $\begin{array}{c}\text { Degrees of } \\
\text { Freedom }\end{array}$ & $\begin{array}{c}\text { Mean } \\
\text { Square }\end{array}$ & F Value \\
\hline Between Quarters & 6061.117 & 3 & 2020.372 & $63.485^{*}$ \\
Residual & 2164.073 & 68 & 31.825 & \\
Total & 8225.190 & 71 & & \\
\hline
\end{tabular}

*Predictable seasonality present at the 99.99 percent confidence level.

tonnage originated increasing from 365 million tons in 1960 to 705 million in 1977, a 93 percent increase. General Freight and "All Other," which collectively accounted for 59 percent of total tonnage in 1977, increased 35 and 158 percent, respectively, over this historical time period. The most rapidly growing component, although small in absolute tonnage, was Refrigerated Solids (primarily Fresh Fruit and Vegetables) which rocketed upward by 315 percent in this time period. Strong tonnage gains were also registered for Heavy Equipment, Building Materials and Agricultural Commodities (principally Wheat, Corn and Other Grain Products).

\section{ECONOMETRIC MODELS}

In building the quarterly econometric models for rail carloadings and heavy duty truck demand, we have linked both rail and truck demand to the major commodity sectors outlined above.

Equation 1 below presents the model of retail sales for Class 8 power units, while Equation 2 highlights the linkages between economic activity and rail transport demand. 


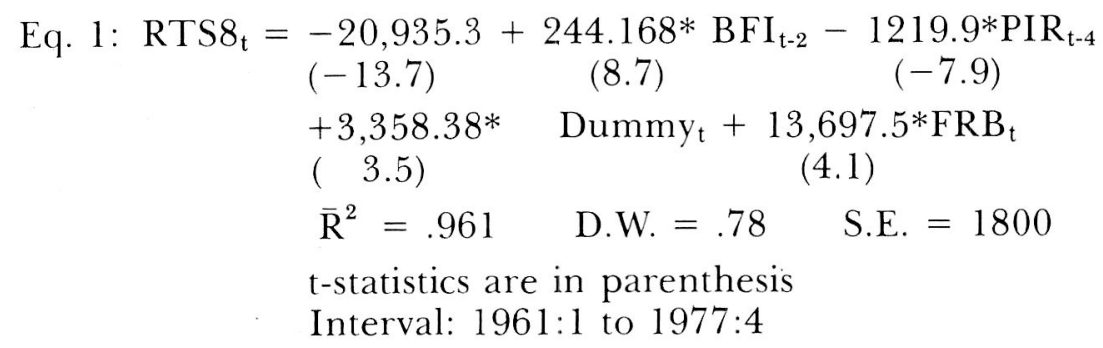

Where: RT S8 = Retail Sales of Heavy Duty Trucks, Seasonally Adjusted.

BFI = Business Fixed Investment in Billions of Constant 1972 Dollars, Seasonally Adjusted Annual Rates.

PIR = Prime Interest Rate.

FRB = Weighted Average of Federal Reserve Board Indexes of Production, Seasonally Adjusted.

$(.15 * \mathrm{FRB} 20+.019 * \mathrm{FRB} 21+.077 * \mathrm{FRB} 22+$ $.095 * \mathrm{FRB} 23+.039 * \mathrm{FRB} 25+.091 * \mathrm{FRB} 26+$ $.134 *$ FRB $27+.064 *$ FRB $30+.229 *$ FRB $36+$ $.06 * \mathrm{FRB} 38+.043 * \mathrm{FRB} 39)$

Where: FRB20 = Index for Food

FRB21 = Index for Tobacco

FRB22 = Index for Textile Mill Products

FRB23 = Index for Apparel

FRB25 = Index for Furniture and Fixtures

FRB26 = Index for Paper and Paper Products

FRB27 = Index for Printing and Publishing

FRB30 = Index for Rubber and Plastic Products

FRB36 = Index for Electric Machinery

FRB38 = Index for Instruments

FRB39 = Index for Miscellaneous Manufacturers.

Dummy $^{1}=$ A Dummy Variable With a Value of Ones in $73: 4$ to $74: 4$, Zeros Elsewhere.

Eq. 2: $\mathrm{FCL}_{\mathrm{t}}=10,479.3+3.049 .13 * \mathrm{FRBMI}_{\mathrm{t}}-7,075.09 *$

$$
\begin{aligned}
& \text { GNPK 72/GNP72 }+ \text { 1,325.61*FRB331/FRB }{ }_{\mathrm{t}}+ \\
& (-11.94) \\
& \text { (8.03) } \\
& 15.2153 * \mathrm{FPI}_{\mathrm{t}} \quad-425.26 * \text { DumStrike }_{\mathrm{t}} \\
& \text { (4.20) } \\
& (-3.0) \\
& \overline{\mathrm{R}}^{2}=.91 \quad \text { D.W. }=1.22 \quad \text { S.E. }=135.5
\end{aligned}
$$


Where: FCL = Freight Carloading-Class 1 Railroads, Adjusted for Capacity and Seasonality.

FRBMI = Federal Reserve Board Index for Mining Products, Seasonally Adjusted.

GNPK72= Potential GNP in Constant 1972 Dollars, Seasonally Adjusted Annual Rates.

GNP72 = GNP in Constant 1972 Dollars, Seasonally Adjusted Annual Rates.

FRB331= Federal Reserve Board Index for Steel Products, Seasonally Adjusted.

FRB = Federal Reserve Board Index for Total Industrial Production, Seasonally Adjusted.

FPI = Farm Proprietor's Income with Inventory Valuation Adjustment and Capital Consumption Adjustment, Seasonally Adjusted.

DumStrike = A Dummy Variable with a Value of One in 71:3, Zeros elsewhere. (Rail Strike)

Retail sales of heavy duty power units are linked to ind ustrial production of eleven key economic sectors which capture the basic composition of commodities transported by the nation's motor carriers, business fixed investment, and the prime interest rate. Truck sales are, as one would expect, positively linked to the output of commodities transported by trucks

TABLE 9

Historical Seasonal Adjustment

Factors: Freight Car Loadings

\begin{tabular}{ccccc}
\hline Year & 1st Qtr. & 2nd Qtr. & 3rd Qtr. & 4th Qtr. \\
\hline 1960 & 93.553 & 103.427 & 101.902 & 101.080 \\
1961 & 93.609 & 103.470 & 101.769 & 101.130 \\
1962 & 93.670 & 103.520 & 101.582 & 101.132 \\
1963 & 93.875 & 103.528 & 101.342 & 101.142 \\
1964 & 94.101 & 123.547 & 101.111 & 101.071 \\
1965 & 94.398 & 103.550 & 100.920 & 100.988 \\
1966 & 94.597 & 103.620 & 100.813 & 100.786 \\
1967 & 94.784 & 103.787 & 100.712 & 100.469 \\
1968 & 95.001 & 104.076 & 100.524 & 100.090 \\
1969 & 95.293 & 104.367 & 100.290 & 99.739 \\
1970 & 95.683 & 104.476 & 100.101 & 99.482 \\
1971 & 96.111 & 104.350 & 100.046 & 99.299 \\
1972 & 96.560 & 104.039 & 100.036 & 99.281 \\
1973 & 96.869 & 103.698 & 100.122 & 99.271 \\
1974 & 97.061 & 103.444 & 100.248 & 99.221 \\
1975 & 97.128 & 103.351 & 100.390 & 99.065 \\
1976 & 97.166 & 103.411 & 100.444 & 98.908 \\
1977 & 97.190 & 103.508 & 100.412 & 98.841 \\
\hline
\end{tabular}


TABLE 10

Historical Seasonal Adjustment

Factors: Retail Sales Of

Heavy Duty Trucks

\begin{tabular}{rrrrr}
\hline Year & 1st Qtr. & 2nd Qtr. & 3rd Qtr. & 4th Qtr. \\
\hline 1960 & 96.812 & 126.795 & 93.900 & 82.370 \\
1961 & 97.172 & 126.503 & 93.693 & 82.551 \\
1962 & 98.099 & 125.257 & 93.590 & 82.981 \\
1963 & 99.684 & 122.996 & 93.614 & 83.800 \\
1964 & 101.286 & 120.563 & 93.325 & 85.125 \\
1965 & 102.652 & 118.156 & 93.085 & 86.583 \\
1966 & 103.480 & 116.097 & 92.985 & 88.136 \\
1967 & 103.703 & 114.350 & 93.115 & 89.799 \\
1968 & 103.126 & 113.259 & 93.270 & 91.349 \\
1969 & 102.234 & 112.479 & 93.580 & 92.656 \\
1970 & 101.345 & 111.620 & 94.178 & 93.809 \\
1971 & 100.440 & 110.644 & 94.962 & 95.002 \\
1972 & 99.438 & 109.548 & 95.964 & 96.033 \\
1973 & 98.488 & 108.537 & 96.971 & 96.748 \\
1974 & 97.792 & 107.738 & 97.769 & 97.223 \\
1975 & 97.260 & 107.270 & 98.329 & 97.399 \\
1976 & 97.007 & 107.030 & 98.613 & 97.451 \\
1977 & 96.943 & 106.872 & 98.757 & 97.435 \\
\hline
\end{tabular}

TABLE 11

Historical Freight Car Loadings

Adjusted For Capacity And

Seasonal Variation

(Millions of Car Loadings)

\begin{tabular}{ccccc}
\hline Year & 1st Qtr. & 2nd Qtr. & 3rd Qtr. & 4th Qtr. \\
\hline 1961 & 6888 & 7037 & 7434 & 7495 \\
1962 & 7515 & 7363 & 7225 & 7212 \\
1963 & 7240 & 7565 & 7439 & 7483 \\
1964 & 7581 & 7624 & 7722 & 7987 \\
1965 & 7851 & 7867 & 7936 & 7940 \\
1966 & 8201 & 8172 & 8282 & 8240 \\
1967 & 8134 & 7951 & 7897 & 8060 \\
1968 & 8121 & 8216 & 8256 & 8199 \\
1969 & 8205 & 8324 & 8442 & 8649 \\
1970 & 8156 & 8308 & 8249 & 8163 \\
1971 & 8248 & 8036 & 7640 & 7168 \\
1972 & 8022 & 8070 & 8274 & 8528 \\
1973 & 8606 & 8554 & 8747 & 8807 \\
1974 & 8795 & 8563 & 8395 & 7993 \\
1975 & 7790 & 7528 & 7506 & 7802 \\
1976 & 8027 & 7818 & 7729 & 7583 \\
1977 & 8139 & 8135 & 7836 & 7546 \\
\hline
\end{tabular}


and business fixed investment, and are negatively influenced by the prime rate.

The dampening effect of the prime rate on retail sales is due to the fact that some 30 percent of industry sales are financed by the manufacturer with the financing charges generally being an "add on" to the prime rate.

The dummy variable in Equation 1 was designed to capture the "prebuy" effect on the part of motor carriers to escape the federally mandated Anti-Lock, Anti-Skid brake system (SS-121) imposed upon truck and trailer manufacturers as of January $1,1975 .^{1}$

Freight car demand is positively linked to the Federal Reserve Board Index of Industrial Production for Mining - a variable which captures the output of coal, iron ore, nonmetallic minerals as well as components of Chemical Products. In 1978, iron ore, coal and nonmetallic minerals collectively accounted for 45 percent of total tons originated by Class 1 Railroads. Rail transport demand is also influenced by output in the primary metals industries as well as farm proprietor income, an excellent proxy for Farm Products (STCC 01) tonnage. Additionally, there is an important interrelationship between rail freight demand and demand for other modes (particularly truck) which is captured by GNPGAP (the ratio of potential to actual GNP). The greater the gap between potential and actual GNP, all other things being held constant, the lower is rail freight demand. In other words, during periods when actual GNP is far below

TABLE 12

Historical Retail Sales of

Heavy Duty Trucks

Adjusted For

Seasonal Variation

\begin{tabular}{rrrrr}
\hline Year & 1st Qtr. & 2nd Qtr. & 3rd Qtr. & 4th Qtr. \\
\hline 1960 & 8,621 & 8,091 & 6,854 & 6,247 \\
1961 & 5,957 & 6,865 & 7,123 & 7,518 \\
1962 & 8,482 & 9,605 & 11,331 & 11,642 \\
1963 & 11,425 & 12,197 & 12,405 & 14,379 \\
1964 & 15,061 & 15,324 & 14,665 & 19,958 \\
1965 & 15,621 & 16,067 & 18,325 & 19,068 \\
1966 & 20,393 & 20,053 & 21,127 & 22,452 \\
1967 & 19,303 & 18,331 & 16,903 & 17,326 \\
1968 & 18,090 & 19,496 & 20,565 & 21,922 \\
1969 & 23,716 & 24,585 & 25,720 & 24,891 \\
1970 & 23,701 & 22,673 & 21,750 & 22,663 \\
1971 & 22,719 & 24,188 & 25,573 & 26,101 \\
1972 & 28,340 & 30,305 & 32,148 & 35,471 \\
1973 & 37,501 & 39,088 & 37,844 & 39,806 \\
1974 & 39,230 & 39,056 & 37,796 & 30,997 \\
1975 & 24,730 & 19,841 & 19,232 & 19,404 \\
1976 & 20,109 & 22,692 & 27,992 & 26,563 \\
1977 & 33,873 & 37,264 & 34,508 & 34,791 \\
\hline
\end{tabular}


TABLE 13

Composition of Class 1 Railroad Tonnage

Originations By Two-Digit STCC Code: 1964-1978

(Millions of Tons)

\begin{tabular}{|c|c|c|c|c|c|c|c|c|c|}
\hline STCC & 1964 & 1967 & 1970 & 1973 & 1974 & 1975 & 1976 & 1977 & 1978 \\
\hline 01 & 131.43 & 123.01 & 134.18 & 156.10 & 142.16 & 134.38 & 133.25 & 121.90 & 128.66 \\
\hline 10 & 116.23 & 108.97 & 126.66 & 125.08 & 126.51 & 106.44 & 96.75 & 83.20 & 112.49 \\
\hline 11 & 357.68 & 384.38 & 404.62 & 376.08 & 390.87 & 407.57 & 397.06 & 414.90 & 383.11 \\
\hline 14 & 182.81 & 170.45 & 163.35 & 170.47 & 171.02 & 150.59 & 133.30 & 139.58 & 134.73 \\
\hline 20 & 94.98 & 103.24 & 110.07 & 106.01 & 107.30 & 100.22 & 99.35 & 98.70 & 95.36 \\
\hline 24 & 85.43 & 91.91 & 101.90 & 108.90 & 105.32 & 88.58 & 99.37 & 99.26 & 95.08 \\
\hline 26 & 32.97 & 37.19 & 42.50 & 46.49 & 48.17 & 40.60 & 43.43 & 44.91 & 41.42 \\
\hline 28 & 65.86 & 81.85 & 91.64 & 99.69 & 101.43 & 91.40 & 98.76 & 104.27 & 106.69 \\
\hline 29 & 30.03 & 28.00 & 36.27 & 52.60 & 53.02 & 45.77 & 44.69 & 46.12 & 44.38 \\
\hline 32 & 70.96 & 77.03 & 71.09 & 72.62 & 67.97 & 56.82 & 56.16 & 59.91 & 59.94 \\
\hline 33 & 83.18 & 83.72 & 82.20 & 71.43 & 70.32 & 51.38 & 49.48 & 55.16 & 60.13 \\
\hline 34 & 9.29 & 13.32 & 11.28 & 10.65 & 11.28 & 8.24 & 5.93 & 2.89 & 2.51 \\
\hline 37 & 20.33 & 23.76 & 24.17 & 34.27 & 29.82 & 27.31 & 29.95 & 33.59 & 32.23 \\
\hline 40 & 35.25 & 36.51 & 39.81 & 44.66 & 48.14 & 38.23 & 35.09 & 36.12 & 37.78 \\
\hline 44 & 4.75 & 4.82 & 4.71 & 4.31 & 4.00 & 3.22 & 3.34 & 4.29 & 4.27 \\
\hline 45 & 2.41 & 3.10 & 4.29 & 6.42 & 6.80 & 6.10 & 6.49 & 8.55 & 9.01 \\
\hline 46 & 7.28 & 8.88 & 10.60 & 20.00 & 21.11 & 17.42 & 19.26 & 22.33 & 24.31 \\
\hline \multicolumn{10}{|l|}{ All } \\
\hline Other & 23.80 & 27.50 & 25.60 & 26.40 & 25.50 & 20.70 & 18.50 & 19.00 & 18.07 \\
\hline Total & 1354.60 & 1407.60 & 1484.90 & 1532.20 & 1530.70 & 1395.00 & 1370.20 & 1394.70 & 1390.17 \\
\hline
\end{tabular}

Source: Interstate Commerce Commission, Freight Commodity Statistics-Class 1 Railroads 
TABLE 13-A

Standard Transportation

Commodity Code

$\begin{array}{ll}\text { STCC } & \text { DESCRIPTION } \\ 01 & \text { Farm Products } \\ 10 & \text { Metallic Ores } \\ 11 & \text { Coal } \\ 14 & \text { Non-metallic Minerals; except fuel } \\ 20 & \text { Food or Kindred Products } \\ 24 & \text { Lumber or Wood Products } \\ 26 & \text { Pulp, Paper or Allied Products } \\ 28 & \text { Chemicals or Allied Products } \\ 29 & \text { Petroleum or Coal Products } \\ 32 & \text { Clay, Concrete, Glass or Stone Products } \\ 33 & \text { Primary Metal Products } \\ 34 & \text { Fabricated Metal Products } \\ 37 & \text { Transportation Equipment } \\ 40 & \text { Waste or Scrap Materials } \\ 44 & \text { Freight Forward Traffic } \\ 45 & \text { Shipper Association } \\ 46 & \text { Miscellaneous Mixed Shipment } \\ \text { Other } & \text { Forest Products (STCC 08), Fresh Fish or Other Marine Products (STCC } \\ & \text { 09), Crude Petroleum \& Natural Gas (STCC 13), Ordnance (STCC 19), } \\ & \text { Tobacco Products (STCC 21), Textile Products (STCC 22), Apparel Prod- } \\ & \text { ucts (STCC 23), Furniture or Fixtures (STCC 25), Printed Matter (STCC } \\ & \text { 27), Rubber or Misc. Plastic Products (STCC 30), Leather Products (STCC } \\ & \text { 31), Non-electric Machinery (STCC 35), Electric Machinery (STCC 36), } \\ & \text { Instruments or Photographic Goods (STCC 39), Miscellaneous Freight } \\ & \text { Shipments (STCC 41), Empty Shipping Containers (STCC 42), Mail \& } \\ \text { Express Traffic (STCC 43). }\end{array}$

potential GNP, the trucking industry (as well as other modes) has excess capacity. The trucker who is an owner-operator is not constrained by regulatory agencies; he reacts quickly during cyclical swings in economic activity and begins to take business away from the rail sector which is devoid of any cyclical pricing strategy. By the same token, motor carriers' marketing and pricing strategies become relatively less important as the economy approaches potential GNP. This phenomenon is clearly demonstrated in regression equations for the truck sector where the ratio of potential to actual GNP has a very significant and positive coefficient. ${ }^{2}$ From the standpoint of its competitive position vis-a-vis other traffic modes, therefore, the rail sector clearly benefits from policy actions promoting full employment. It was also recognized by the Task Force on Railroad Productivity that the railroads need an aggressive marketing policy ${ }^{3}$; Equation 2 suggests the need is particularly acute during periods of recession.

\section{FORECASTING PERFORMANCE}

To test the forecasting accuracy of each model, both were estimated over 
TABLE 14

Class 1 and 2 Motor Carrier Tonnage

Originated By Commodity Category

1960-1977

(Millions of Tons)

\begin{tabular}{|c|c|c|c|c|c|c|c|c|c|c|}
\hline & 1960 & 1965 & 1970 & 1971 & 1972 & 1973 & 1974 & 1975 & 1976 & 1977 \\
\hline General Freight & 163.4 & 210.1 & 217.9 & 221.1 & 247.2 & 257.1 & 234.9 & 200.1 & 211.1 & 220.5 \\
\hline Household Goods & 2.3 & . $\quad 2.4$ & 6.2 & 2.9 & 2.8 & 2.8 & 2.9 & 2.6 & 2.6 & 2.9 \\
\hline Heavy Machinery & 2.7 & 4.2 & 6.0 & 6.5 & 7.4 & 8.5 & 9.1 & 7.9 & 8.8 & 10.5 \\
\hline Liquid Petroleum & 87.7 & 125.3 & 156.3 & 155.3 & 169.3 & 195.1 & 174.9 & 161.2 & 160.9 & 185.4 \\
\hline Refrigerated Liquids & 1.2 & 1.8 & 1.9 & .9 & 1.0 & .9 & .6 & $.6)$ & & \\
\hline Refrigerated Solids & 4.0 & 6.3 & 10.0 & 10.8 & 12.5 & 17.7 & 17.3 & 10.3) & 20.7 & 21.6 \\
\hline Agricultural Commodities & 7.1 & 7.2 & 8.6 & 9.5 & 11.1 & 12.8 & 11.8 & 11.7 & 12.6 & 13.9 \\
\hline Motor Vehicles & 14.0 & 19.4 & 15.7 & 22.1 & 24.3 & 27.7 & 22.1 & 19.9 & 23.7 & 26.9 \\
\hline Building Materials & 7.4 & 18.9 & 24.2 & 28.3 & 29.4 & 26.7 & 23.3 & 21.3 & 29.7 & 40.0 \\
\hline All Other Commodities & 75.2 & 122.5 & 142.5 & 150.2 & 170.2 & 199.2 & 184.7 & 164.9 & 171.4 & 194.2 \\
\hline Total & 365.0 & 518.1 & 589.3 & 607.6 & 675.2 & 738.5 & 681.5 & 608.5 & 649.4 & 705.4 \\
\hline \multicolumn{11}{|c|}{$\%$ Distribution } \\
\hline General Freight & 44.8 & 40.6 & 37.0 & 36.4 & 36.6 & 34.8 & 34.5 & 32.9 & 32.5 & 31.3 \\
\hline Household Goods & .6 & .5 & 1.1 & .5 & .4 & .4 & .4 & .4 & .4 & .4 \\
\hline Heavy Machinery & .7 & .8 & 1.0 & 1.1 & 1.1 & 1.2 & 1.3 & 1.3 & 1.4 & 1.5 \\
\hline Liquid Petroleum & 24.0 & 24.2 & 26.5 & 25.5 & 25.0 & 25.1 & 25.6 & 26.5 & 26.0 & 26.3 \\
\hline Refrigerated Liquids & .3 & .3 & .3 & .1 & .1 & .1 & .1 & $.1)$ & & \\
\hline Refrigerated Solids & 1.1 & 1.2 & 1.7 & 1.8 & 1.9 & 2.4 & 2.5 & $3.0)$ & 3.7 & 3.0 \\
\hline Agricultural Commodities & 1.9 & 1.4 & 1.5 & 1.6 & 1.6 & 1.7 & 1.7 & 1.9 & 1.9 & 2.0 \\
\hline Motor Vehicles & 3.8 & 3.7 & 2.7 & 3.6 & 3.6 & 3.9 & 3.2 & 3.3 & 3.6 & 3.8 \\
\hline Building Materials & 2.0 & 3.6 & 4.1 & 4.7 & 4.4 & 3.6 & 3.4 & 3.5 & 4.6 & 5.7 \\
\hline All Other Commodities & 20.6 & 23.6 & 24.2 & 24.7 & 25.2 & 27.0 & 27.1 & 27.1 & 26.4 & 27.5 \\
\hline Total & 100.0 & 100.0 & 100.0 & 100.0 & 100.0 & 100.0 & 100.0 & 100.0 & 100.0 & 100.0 \\
\hline
\end{tabular}

Source: American Trucking Association, Intercity Truck Tonnage Report (1960-1976) and Motor Carrier Statistical Report (1976-1977), Annual Report. 
TABLE 15

Model Test Results For

Retail Sales Of

Heavy Duty Trucks

Seasonally Adjusted

\begin{tabular}{|c|c|c|c|}
\hline Fitted Period & & $\begin{array}{c}\text { One } \\
\text { Quarter Ahead }\end{array}$ & $\begin{array}{l}\text { Two } \\
\text { Quarters Ahead }\end{array}$ \\
\hline \multicolumn{4}{|l|}{$1961: 1$ to $1977: 4$} \\
\hline & Forecast & 36,908 & 39,226 \\
\hline & Actual & 39,272 & 40,041 \\
\hline & Error & $(2,364)$ & $(815)$ \\
\hline & Percent Error & $(6.1)$ & $(2.0)$ \\
\hline \multicolumn{4}{|l|}{$1961: 1$ to $1978: 1$} \\
\hline & Forecast & 37,861 & 38,209 \\
\hline & Actual & 40,041 & 40,671 \\
\hline & Error & $(2,180)$ & $(2,462)$ \\
\hline & Percent Error & $(\quad 5.4)$ & ( 6.1$)$ \\
\hline \multirow[t]{5}{*}{$1961: 1$ to $1978: 2$} & & & \\
\hline & Forecast & 38,132 & 39,292 \\
\hline & Actual & 40,671 & 41,874 \\
\hline & Error & $(2,539)$ & $(2,582)$ \\
\hline & Percent Error & $(\quad 6.2)$ & $(\quad 6.0)$ \\
\hline \multicolumn{4}{|l|}{$1961: 1$ to $1978: 3$} \\
\hline & Forecast & 40,416 & 41,035 \\
\hline & Actual & 41,874 & 46,262 \\
\hline & Error & $(1,458)$ & $(5,227)$ \\
\hline & Percent Error & ( 3.5$)$ & ( 11.3$)$ \\
\hline \multicolumn{4}{|l|}{ Total } \\
\hline & Forecast & 153,317 & 157,762 \\
\hline & Actual & 161,858 & 168,848 \\
\hline & Error & $(8,541)$ & $(11,086)$ \\
\hline & Percent Error & $(\quad 5.3)$ & $(6.5)$ \\
\hline
\end{tabular}

four successive time frames and used to forecast one and two quarters ahead. Forecasts of the independent variables were obtained from Data Resources, Inc. The values which were used would have been the estimates available at the time the forecast would have been made.

A record of forecasting accuracy is presented in Tables 15 and 16. Forecasting one quarter out, errors without regard to sign ranged from 3.5 percent for heavy duty truck retail sales to 19.4 percent for freight carloadings. Estimating two quarters out, the errors ranged from 2.0 percent for retail sales to 19.0 percent for freight carloadings. A significant portion of the forecasting error for freight carloading in quarters 78:3 and 78:4 resulted from the BRAC rail strike in September. The third quarter experienced depressed freight car movements while the fourth quarter data is biased on the high side due to unusually heavy shipments after the strike was settled. 
TABLE 16

Model Test Results For

Freight Car Loadings

Adjusted For Capacity And

Seasonally Adjusted

\begin{tabular}{|c|c|c|c|}
\hline Fitted Period & & $\begin{array}{c}\text { One } \\
\text { Quarter Ahead }\end{array}$ & $\begin{array}{l}\text { Two } \\
\text { Quarters Ahead }\end{array}$ \\
\hline \multicolumn{4}{|c|}{$1961: 1$ to $1977: 4$} \\
\hline & Forecast & 7,222 & 8,338 \\
\hline & Actual & 6,723 & 7,683 \\
\hline & Error & 499 & 655 \\
\hline & Percent Error & 7.4 & 8.5 \\
\hline \multicolumn{4}{|c|}{$1961: 1$ to $1978: 1$} \\
\hline & Forecast & 8,120 & 8,155 \\
\hline & Actual & 7,683 & 7,494 \\
\hline & Error & 437 & 661 \\
\hline & Percent Error & 5.7 & 8.8 \\
\hline \multicolumn{4}{|c|}{$1961: 1$ to $1978: 2$} \\
\hline & Forecast & 8,264 & 8,295 \\
\hline & Actual & 7,494 & 10,238 \\
\hline & Error & 770 & $(1,943)$ \\
\hline & Percent Error & 10.3 & ( 19.0$)$ \\
\hline \multicolumn{4}{|c|}{$1961: 1$ to $1978: 3$} \\
\hline & Forecast & 8,248 & 8,250 \\
\hline & Actual & 10,238 & 7,960 \\
\hline & Error & $(1,990)$ & 290 \\
\hline & Percent Error & ( 19.4) & 3.6 \\
\hline \multicolumn{4}{|l|}{ Total } \\
\hline & Forecast & 31,854 & 33,038 \\
\hline & Actual & 32,138 & 33,375 \\
\hline & Error & $(284)$ & $(337)$ \\
\hline & Percent Error & $(\quad 0.9)$ & $(\quad 1.0)$ \\
\hline
\end{tabular}

In the aggregate, the models seem to produce forecasts biased on the low side. Percentage errors for the sum of estimates one and two quarters out for Retail Sales were 5.3 percent and 6.5 percent, respectively, under actual values. Aggregate test results for freight carloadings one and two quarters ahead performed 0.9 percent and 1.0 percent under reported car movements. Judgment, which was not applied to the forecasts in Tables 14 and 15, may improve the forecasting ability of each equation.

\section{CONCLUSION}

The models outlined above provide transportation economists with an excellent planning tool for measuring the impact on demand from shortrun fluctuations in the economy. A quantitative linkage between variables external to the freight sector enables decision-makers to focus attention on 
sectors which generate the "derived" demand for modal services and, hence, provide important information in terms of managerial decisions regarding raw material and labor requirement in a short-term planning time horizon.

\section{FOOTNOTES}

${ }^{1}$ For a quantitative analysis of the effect of mandated government safety standards see $\mathrm{H}$. Wade German and Larry C. Peppers, "Anti-Locking Brake Systems: The Impact of Public Safety Standards on the Demand for Truck Trailers," Proceedings, Transportation Research Forum-1976, pp. 412-15.

${ }^{2}$ See H. Wade German and Larry C. Peppers "Social
Control of Business: Implications for Logistics Planning” Robert G. House and James F. Robeson (ed), Interfaces: Logistics, Marketing and Production (Ohio State University-1976)

${ }^{3}$ Task Force on Rail Productivity, Improving Railroad Productivity Washington, D.C., (Government Printing Office, 1973), p. 13. 